\title{
Strong plasmonic enhancement of photovoltage in graphene
}

T.J. Echtermeyer', L. Britnell², P.K. Jasnos', A. Lombardo1, R.V. Gorbachev³, A.N. Grigorenko², A.K. Geim³, A.C. Ferrari ${ }^{1} \&$ K.S. Novoselov²

From the wide spectrum of potential applications of graphene, ranging from transistors and chemical sensors to nanoelectromechanical devices and composites, the field of photonics and optoelectronics is believed to be one of the most promising. Indeed, graphene's suitability for high-speed photodetection was demonstrated in an optical communication link operating at $10 \mathrm{Gbits}^{-1}$. However, the low responsivity of graphene-based photodetectors compared with traditional III-V-based ones is a potential drawback. Here we show that, by combining graphene with plasmonic nanostructures, the efficiency of graphene-based photodetectors can be increased by up to 20 times, because of efficient field concentration in the area of a $p-n$ junction. Additionally, wavelength and polarization selectivity can be achieved by employing nanostructures of different geometries.

${ }^{1}$ Department of Engineering, University of Cambridge, Cambridge CB3 OFA, UK. ${ }^{2}$ School of Physics and Astronomy, University of Manchester, Oxford Road, Manchester M13 9PL, UK. ${ }^{3}$ Centre for Mesoscience and Nanotechnology, University of Manchester, Oxford Road, Manchester M13 9PL, UK. Correspondence and requests for materials should be addressed to K.S.N. (email: kostya@manchester.ac.uk). 
T he unique optical and electronic properties of graphene ${ }^{1,2}$ make it ideal for a number of photonic applications $s^{3-7}$. In particular, graphene-based photodetectors have excellent characteristics in terms of quantum efficiency and reaction time, because of the very large room-temperature mobility and high Fermi velocity of charge carriers in this material ${ }^{8-10}$. Although the exact mechanism for light to current conversion is still debated ${ }^{11,12}$, a $\mathrm{p}-\mathrm{n}$ junction is usually required to separate the photo-generated electron-hole pairs. Such p-n junctions are often created close to the contacts, because of the difference in the work functions of metal and graphene ${ }^{13,14}$. Whatever the photocurrent generation mechanism, all such devices suffer from several problems, such as low light absorption of graphene ( $2.3 \%$ of normal incident light $\left.{ }^{15,16}\right)$; difficulty of extracting photoelectrons (only a small area of the $\mathrm{p}-\mathrm{n}$ junction contributes to current generation); and the absence of a photocurrent for the condition of uniform flood illumination on both contacts of the device. Unless the contacts are made of different materials, the voltage/current produced at both contacts will be of opposite polarity for symmetry reasons, resulting in zero net signal $1^{8,10,11}$.

One possible way of overcoming these restrictions is to utilize plasmonic nanostructures placed near the contacts. Incident light, absorbed by such nanostructures, can be efficiently converted into plasmonic oscillations, which leads to a dramatic enhancement of the local electric field. One might consider this process as generation of evanescent photons that exist only in the near-field region ${ }^{17-20}$. Such a field enhancement, exactly in the area of the $\mathrm{p}-\mathrm{n}$ junction formed in graphene, can result in a significant performance improvement of graphene-based photodetectors. The role of the suggested plasmonic nanostructures is therefore to guide the incident electromagnetic energy directly to the region of the $\mathrm{p}-\mathrm{n}$ junction. Here we demonstrate that the efficiency of nanostructured devices of this kind can be 20 times larger than that of traditional devices ${ }^{8-10}$.

\section{Results}

Sample design. We used graphene prepared by micromechanical exfoliation of graphite ${ }^{21,22}$. The single-layer nature of our flakes was confirmed by a combination of optical contrast ${ }^{23-25}$, Raman spectroscopy ${ }^{26}$ and quantum Hall effect ${ }^{27,28}$ measurements. Ti/Au $(3 \mathrm{~nm} \mathrm{Ti}, 80 \mathrm{~nm} \mathrm{Au})$ contacts were formed by e-beam lithography, e-beam evaporation and lift-off. Figure 1a shows the layout of the resulting devices. Various nanostructures were fabricated close to the macroscopic contacts of such two-terminal devices (examples are shown in Fig. 1b-d). The layout and composition of the structures are chosen to produce strong light absorption in the visible range, and are similar to what we previously designed to achieve a plasmonic blackbody, resulting in almost complete absorption of incident visible light ${ }^{29}$. We employed several different designs, but here we will mainly concentrate on one particular structure (grating with $110 \mathrm{~nm}$ finger width, $300 \mathrm{~nm}$ pitch, Fig. 1b), which showed the best performance.

Electrical characterization. The local photovoltage and photocurrent response of our devices is measured by coupling several lasers to a microscope, as described in Methods, and by scanning the position of the illumination spot. This allows us to measure the photovoltage dependence on intensity, wavelength and polarization of the incoming light, as well as the gate voltage. The laser power on the samples is kept at $\sim 30 \mu \mathrm{W}$. At this power, the photovoltage signal is larger than any thermopower-related signal (verified by changing the incident power). This laser intensity is also low enough not to result in any observable overheating of the samples (which ensures that we work in the linear regime).

Our devices have reasonable field effect mobility $5,000 \mathrm{~cm}^{2} \mathrm{~V}^{-1} \mathrm{~s}^{-1}$ at room temperature (Fig. 2a). They show unintentional p-doping of up to $5 \times 10^{12} \mathrm{~cm}^{-2}$ (confirmed both by electronic transport ${ }^{30}$ and Raman measurements ${ }^{31,32}$, Fig. $\left.2 c, d\right)$, probably because of water
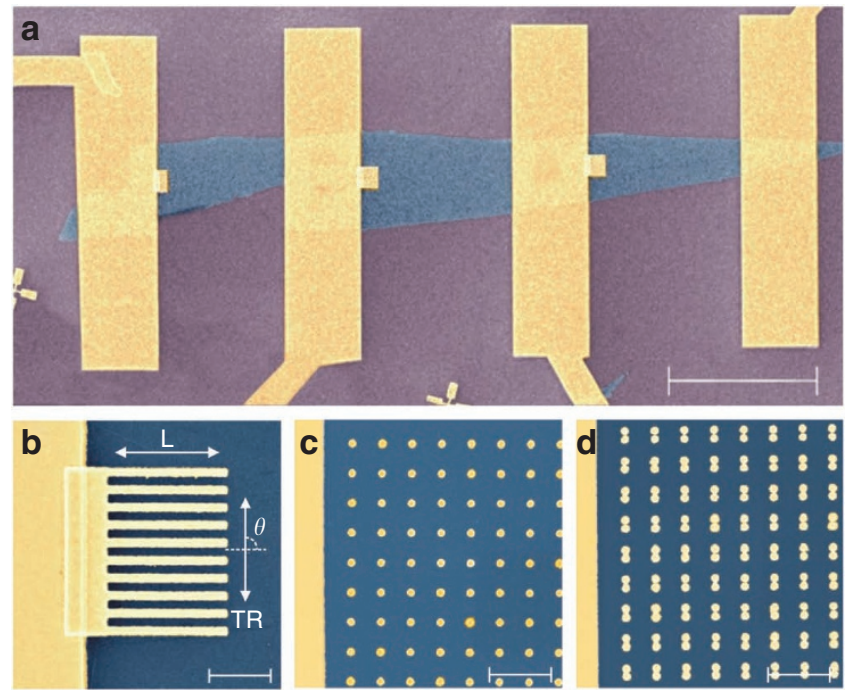

Figure 1 | Scanning electron microscopy micrographs of the graphene devices with plasmonic nanostructures. (a) An overall image of one of our devices (in false colours). Blue, graphene; purple, $\mathrm{SiO}_{2}(300 \mathrm{~nm})$; yellow, $\mathrm{Ti} / \mathrm{Au}$ electrodes. Scale bar, $20 \mu \mathrm{m}$. (b-d) Blow up of contacts with various tested plasmonic nanostructures (in false colours). L and TR incident light polarizations are indicated. Scale bar, $1 \mu \mathrm{m}$. a

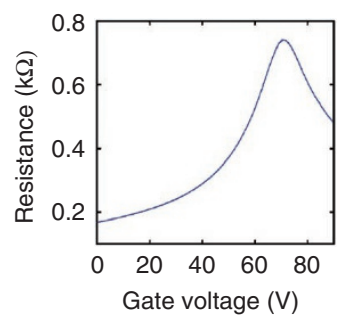

b

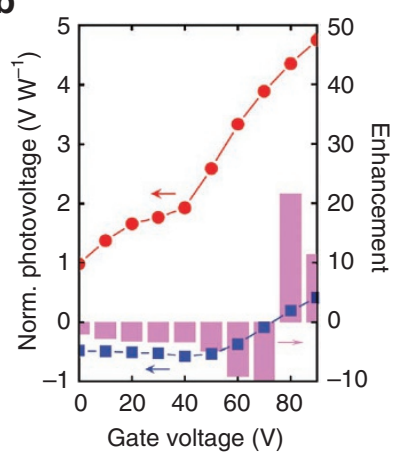

C

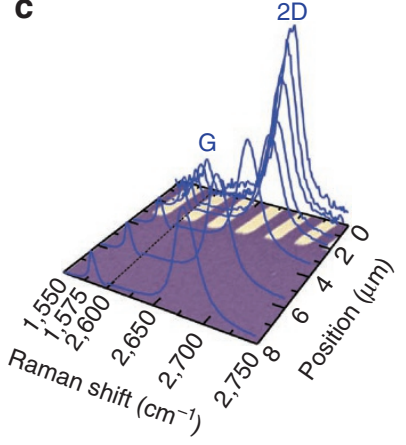

d

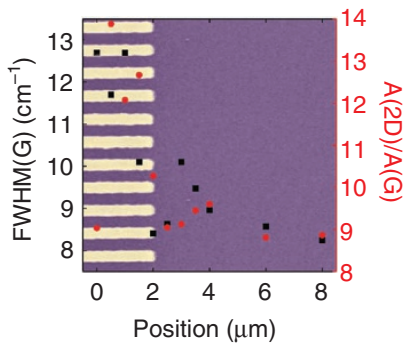

Figure $\mathbf{2}$ | Resistance and photovoltaic characteristics. (a) Resistance as function of the gate voltage. (b) Normalized photovoltage for illumination close to the FC (blue), close to the SC (red) and enhancement (purple) as function of the gate voltage. Illumination wavelength $514 \mathrm{~nm}$. (c) Raman spectra recorded on graphene at different distances from the SC. (d) $F W H M(G)$ and $A(2 D) / A(G)$ as a function of position.

adsorption $^{30}$. The contacts provide local weak p-type doping ${ }^{14}$, again confirmed by the Raman data, Figure $2 c$,d. The photovoltage generated on the non-structured, flat part of the contact (FC), is positive 

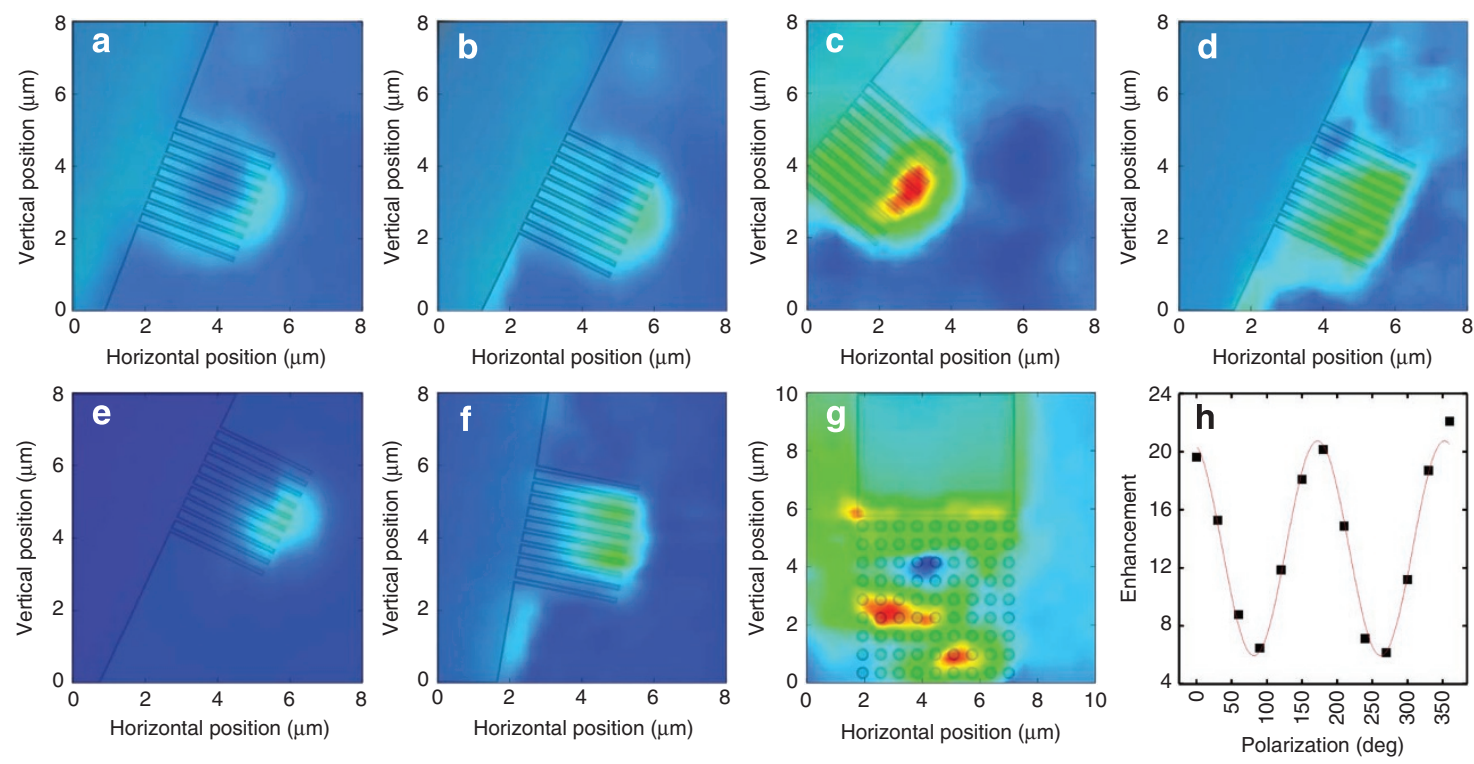

Figure $\mathbf{3}$ | Photovoltage maps of one of the nanostructured contacts. The signal (normalized to laser power) measured on a contact with finger structure (finger width $110 \mathrm{~nm}$, pitch $300 \mathrm{~nm}$, except for $\mathbf{g}$ ) as a function of the position of the illumination spot (spot size $1.5 \mu \mathrm{m}$ for various excitation wavelengths. Gate voltage $90 \mathrm{~V}$. Colour scale (except for $\mathbf{g}$ ), from $0 \mu \mathrm{V}$ (blue) to $20 \mu \mathrm{V}$ (red). Overlaid is a schematic position of the contact. (a) $457 \mathrm{~nm}$, TR polarization. (b) $488 \mathrm{~nm}$, TR polarization. (c) $514 \mathrm{~nm}$, TR polarization. (d) $633 \mathrm{~nm}$, TR polarization. (e) $785 \mathrm{~nm}$, TR polarization. (f) $514 \mathrm{~nm}$, L polarization. (g) Example of photovoltage measured on a sample with an array of nanodots; $633 \mathrm{~nm}$, TR polarization. Colour scale, from $-4 \mu \mathrm{V}$ (blue) to $12 \mu \mathrm{V}$ (red). (h) Polarization-dependent enhancement at $\lambda=514 \mathrm{~nm}$, with $0^{\circ}$ being TR polarization. Black squares, measured data; red line, $\cos ^{2} \theta$ fit.

for electron doping, and negative for hole doping, as a consequence of the formation of $\mathrm{p}-\mathrm{n}$ or $\mathrm{p}^{-}-\mathrm{p}^{+}$junctions, see Figure $2 \mathrm{~b}$.

Photovoltage measurements. The photovoltage generated on the structured part of the contact (SC) is significantly higher than that on the FC. The enhancement is more than one order of magnitude for the case of the $\mathrm{p}-\mathrm{n}$ junction (Fig. 2b). However, the photovoltage generated on the SC has remarkably different behaviour than that on the FC. It is positive for all the gate voltages measured, monotonically decreasing for higher hole doping, Figure $2 \mathrm{~b}$. We do not have a complete understanding of this phenomenon, but we speculate that the most probable reason is the complex distribution of the optical electric field around the SC, which allows us to probe different parts of the $\mathrm{p}-\mathrm{n}$ or $\mathrm{p}^{-}-\mathrm{p}^{+}$junctions in comparison with the FC.

The doping profile is confirmed by a Raman line scan across the contacts, carried out at zero gate voltage, Figure $2 \mathrm{c}$. Figure $2 \mathrm{~d}$ plots the ratios of the areas of $2 \mathrm{D}$ and $\mathrm{G}$ peaks, $\mathrm{A}(2 \mathrm{D}) / \mathrm{A}(\mathrm{G})$, and the full width at half maximum of the G peak, FWHM(G). Far away from the contacts, the Raman parameters correspond to $\sim 5 \times 10^{12} \mathrm{~cm}^{-2}$ p-doping ${ }^{31,32}$ (Fig. 2d), consistent with the transport gate-voltage measurements (Fig. 2a). A(2D)/A(G) significantly increases when moving close to the contacts, accompanied by a FWHM(G) increase. This implies the sample becomes less p-doped, with the area around contacts being only lightly p-doped, up to about few $10^{11} \mathrm{~cm}^{-2}$ (refs $31,32)$. In the vicinity of the SC, it can be seen in Figure $2 \mathrm{~d}$ that both FWHM $(\mathrm{G})$ and $\mathrm{A}(2 \mathrm{D}) / \mathrm{A}(\mathrm{G})$ exhibit a non-monotonous behaviour, resulting in local maxima. This can be explained by the interplay between the inhomogeneous doping and strong amplification of the Raman signal around the metallic nanostructures ${ }^{18}$.

Photovoltage mapping and polarization dependence. To demonstrate the plasmonic nature of the enhancement, we mapped the photovoltaic response for different polarizations and excitation wavelengths for normal light incidence (Fig. 3). This allowed us to directly compare the signal produced when shining light on the FC and SC. Wavelengths covering the visible to near-infrared range
(457, 488, 514, 633 and $785 \mathrm{~nm}$, corresponding to Fig. 3a-e, respectively) were used. Figure 3 shows that the SC provides some level of enhancement for all the wavelengths used (the photovoltage on the $\mathrm{SC}$ is always larger than that on the FC). The generated photovoltage is usually maximum when the laser beam is positioned at the tips of the nanostructures. This is because, in this area, both large electron band bending (because of doping from the contacts ${ }^{13,14}$ ) and strong enhancement of the optical field ${ }^{17-20}$ are achieved. In between the metal stripes, although the optical field enhancement is still produced, the band bending is significantly smaller because of screening by the metal contacts.

We observed enhancement of the photovoltage for all wavelengths that we measured, with maximum amplification of more than 20 at the plasmonic resonance of our structures. Indeed, the strong spectral dependence of the photovoltaic enhancement suggests the importance of the plasmonic resonances in our nanostructures. The maximum enhancement for $110 \mathrm{~nm}$ wide stripes (Figs. 3 and 4) is observed at $514 \mathrm{~nm}$ (Fig. 4a, which is useful for solar cell applications, for instance). Depending on the SC dimensions, the resonance can be tailored to match any part of the spectrum, which might be important for applications in telecommunication. Indeed, for wider structures, the resonance shifts towards larger wavelengths (for example, the $130 \mathrm{~nm}$ wide stripes have maximum enhancement close to $633 \mathrm{~nm}$, Fig. 4b). Such wavelength dependence rules out the possibility that this enhancement is simply because of the geometric enlargement of the junction area for the nanostructured contacts. We note that light interference in $\mathrm{SiO}_{2}$ can provide some dependence of the photovoltage on the wavelength of the excited light, and can be used to enhance the signal even further ${ }^{33}$. However, in our experiments, the enhancement coefficient (Fig. 4) does not depend on the optical properties of $\mathrm{SiO}_{2}$ and allows us to concentrate on the performance of such plasmonic nanostructures.

The SC photovoltage polarization dependence can be fitted with a $\cos ^{2} \theta$ function, Figure $3 \mathrm{~h}$, where $\theta$ is the angle between the polarization and the long sides of the nanostructured 'fingers' (Fig. 1). The transverse (TR) polarization gives much stronger enhancement 

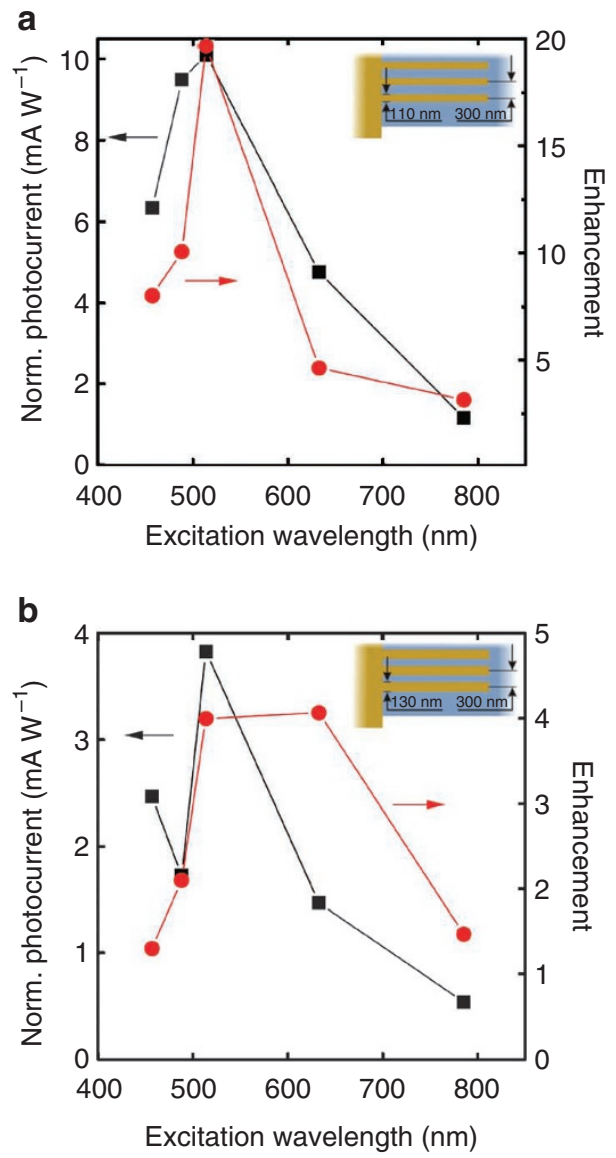

Figure 4 | Normalized photocurrent and maximum enhancement coefficient. Both characteristics measured as a function of excitation wavelength for two of our finger structures of $300 \mathrm{~nm}$ pitch and (a) finger width $110 \mathrm{~nm}$ and (b) $130 \mathrm{~nm}$. Insets, schematic representations of such structures.

than the longitudinal (L) polarization, as the former couples resonantly to the plasmonic modes across the nanostructured fingers, matching the plasmon wavelength ${ }^{34}$. The FC photovoltage polarization dependence is much weaker (the difference between TR and $\mathrm{L}$ does not exceed $30 \%$ ). We stress that, although the far-field polarization properties of the metal stripes also show $\cos ^{2} \theta$ dependence, they cannot be responsible for the enhancement of photovoltage compared with the FC. Hence the observed large anisotropy in enhancement ratio comes from the near-fields generated by plasmonic nanoresonators.

\section{Discussion}

We modelled the enhancement of the electric field with the help of finite element analysis using the High Frequency Structure Simulator $(\text { HFSS11) })^{35}$. The actual device geometry was utilized in the model, and the optical constants of gold, graphene and the substrate were taken from ref. 29. Figure 5 shows the amplitude of the in-plane electric field around the nanostructures for incident light wavelengths of $514 \mathrm{~nm}$ (Fig. 5a,b) and $633 \mathrm{~nm}$ (Fig. 5b,c) and TR and L polarizations. The results correlate well with our experimental data, see Figures 3 and 4 . Thus, the TR polarization for $514 \mathrm{~nm}$ excitation (Fig. 5a) gives very strong field enhancement on $110 \mathrm{~nm}$ wide structures: a factor 5 in terms of field, which is a factor 25 in terms of power amplification-very similar to what we observe in our experiment, Figure 4. The enhancement is much weaker for $633 \mathrm{~nm}$ excitation, again in excellent agreement with our experiments. We note, how-
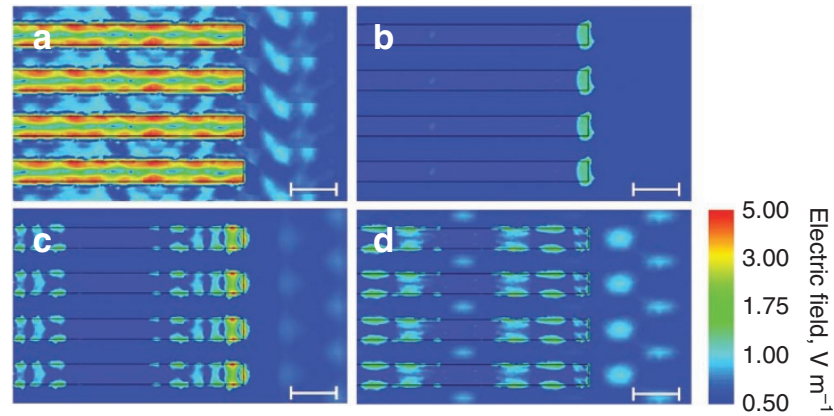

Figure $\mathbf{5}$ | Numerical finite element simulations of local electric field. (a) $514 \mathrm{~nm}$, TR polarization. (b) $514 \mathrm{~nm}$, L polarization. (c) $633 \mathrm{~nm}, \mathrm{TR}$ polarization. (d) $633 \mathrm{~nm}$, L polarization. Scale bar, $300 \mathrm{~nm}$.

ever, that one cannot draw a direct quantitative comparison between the calculated field enhancement and the measured photovoltaic signals. Indeed, the generated photovoltage depends on two factors: the amplitude of the local optical field and the strength and direction of the electronic band bending (built-in electric field because of the $\mathrm{p}-\mathrm{n}$ junctions). The field amplification is strongly inhomogeneous, increasing strongly near the contact edges, Figure 5a. This, together with the fact that the $\mathrm{p}-\mathrm{n}$ junction profile might also be non-trivial, complicates the problem. However, the qualitative correspondence between the experimental results and the theoretical predictions proves the viability of the concept of using field amplification by plasmonic nanostructures for light harvesting in graphene-based photonic devices.

As an additional benefit, nanostructures with geometries resonant at desired wavelengths can be utilized in graphene-based photodetectors for selective amplification, potentially allowing light filtering and detection, as well as polarization determination in a single device at high operating frequencies. The frequency performance can be even improved in comparison with traditional devices, as the plasmonic structures add only negligible contribution to the capacitance (fractions of femto-Farads), but can significantly reduce the contact resistance. We believe that further optimization of such plasmonic nanostructures (for example, making use of coupled or cascaded plasmon resonances ${ }^{35,36}$ ) might lead to even greater photovoltage enhancement.

\section{Methods}

Device fabrication. Graphene was exfoliated from graphite by mechanical cleavage onto $\mathrm{Si}$ covered with $300 \mathrm{~nm} \mathrm{SiO}{ }_{2}$. Subsequently, contacts including nanostructures were fabricated using e-beam lithography, $\mathrm{Ti}(3 \mathrm{~nm}) / \mathrm{Au}(80 \mathrm{~nm})$ evaporation and lift-off. Fabricated samples were bonded in a chip carrier for characterization.

Photovoltage mapping. A 2182A Nanovoltmeter (Keithley) was used to record the photovoltage at the device terminals with an additional 2400 Sourcemeter (Keithley), allowing control of the gate voltage. Laser light (457, 488, 514, 633 and $785 \mathrm{~nm}$ ) generated from multiwavelength $\mathrm{Ar}+, \mathrm{He}-\mathrm{Ne}$ and solid-state infrared lasers was coupled to the sample by using a DM LM microscope (Leica) and a $\times 100$ ultra-long working distance objective, resulting in a $\sim 1.5-\mu \mathrm{m}$ spot size. A Physik Instrumente piezoelectric stage was used to translate the sample with respect to the laser spot in the $\mathrm{x} / \mathrm{y}$ directions with $200-\mathrm{nm}$ steps, resulting in position-dependent recording of the generated photovoltage. Measurements were done at room temperature in ambient atmosphere.

Raman spectroscopy. Raman spectra were collected by coupling the light scattered from the sample to an inVia Raman spectrometer (Renishaw). Spectra are recorded by using a DM LM microscope (Leica) using a $\times 100$ ultra-long working distance objective. The laser power is kept below $1.5 \mathrm{~mW}$ to exclude possible heating effects.

\section{References}

1. Geim, A. K. \& Novoselov, K. S. The rise of graphene. Nat. Mater. 6, 183-191 (2007). 
2. Geim, A. K. Graphene: status and prospects. Science 324, 1530-1534 (2009).

3. Bonaccorso, F., Sun, Z., Hasan, T. \& Ferrari, A. C. Graphene photonics and optoelectronics. Nat. Photonics 4, 611-622 (2010).

4. Blake, P. et al. Graphene-based liquid crystal device. Nano Lett. 8, 1704-1708 (2008).

5. Wang, X., Zhi, L. J. \& Mullen, K. Transparent, conductive graphene electrodes for dye-sensitized solar cells. Nano Lett. 8, 323-327 (2008).

6. Liu, M. et al. A graphene-based broadband optical modulator. Nature 474, 64-67 (2011).

7. Sun, Z. P. et al. Graphene mode-locked ultrafast laser. ACS Nano 4, 803-810 (2010).

8. Lee, E. J. H., Balasubramanian, K., Weitz, R. T., Burghard, M. \& Kern, K. Contact and edge effects in graphene devices. Nat. Nanotechnol. 3, 486-490 (2008).

9. Xia, F. N., Mueller, T., Lin, Y. M., Valdes-Garcia, A. \& Avouris, P. Ultrafast graphene photodetector. Nat. Nanotechnol. 4, 839-843 (2009).

10. Mueller, T., Xia, F. N. A. \& Avouris, P. Graphene photodetectors for high-speed optical communications. Nat. Photonics 4, 297-301 (2010).

11. Park, J., Ahn, Y. H. \& Ruiz-Vargas, C. Imaging of photocurrent generation and collection in single-layer graphene. Nano Lett. 9, 1742-1746 (2009).

12. Xu, X. D., Gabor, N. M., Alden, J. S., van der Zande, A. M. \& McEuen, P. L. Photo-thermoelectric effect at a graphene interface junction. Nano Lett. 10, 562-566 (2010).

13. Giovannetti, G. et al. Doping graphene with metal contacts. Phys. Rev. Lett. 101, 026803 (2008).

14. Blake, P. et al. Influence of metal contacts and charge inhomogeneity on transport properties of graphene near the neutrality point. Solid State Commun. 149, 1068-1071 (2009).

15. Nair, R. R. et al. Fine structure constant defines visual transparency of graphene. Science 320, 1308 (2008)

16. Kuzmenko, A. B., van Heumen, E., Carbone, F. \& van der Marel, D. Universal optical conductance of graphite. Phys. Rev. Lett. 100, 117401 (2008)

17. Nie, S. M. \& Emery, S. R. Probing single molecules and single nanoparticles by surface-enhanced Raman scattering. Science 275, 1102-1106 (1997).

18. Schedin, F. et al. Surface-enhanced Raman spectroscopy of graphene. ACS Nano 4, 5617-5626 (2010).

19. Lee, J., Novoselov, K. S. \& Shin, H. S. Interaction between metal and graphene: dependence on the layer number of graphene. ACS Nano 5, 608-612 (2011).

20. Grigorenko, A. N., Roberts, N. W., Dickinson, M. R. \& Zhang, Y. Nanometric optical tweezers based on nanostructured substrates. Nat. Photonics 2, 365-370 (2008)

21. Novoselov, K. S. et al. Electric field effect in atomically thin carbon films. Science 306, 666-669 (2004).

22. Novoselov, K. S. et al. Two-dimensional atomic crystals. Proc. Natl Acad. Sci. USA 102, 10451-10453 (2005).

23. Blake, P. et al. Making graphene visible. Appl. Phys. Lett. 91, 063124 (2007).

24. Abergel, D. S. L., Russell, A. \& Fal'ko, V. I. Visibility of graphene flakes on a dielectric substrate. Appl. Phys. Lett. 91, 063125 (2007).

25. Casiraghi, C. et al. Rayleigh imaging of graphene and graphene layers. Nano Lett. 7, 2711-2717 (2007).
26. Ferrari, A. C. et al. Raman spectrum of graphene and graphene layers. Phys. Rev. Lett. 97, 187401 (2006)

27. Novoselov, K. S. et al. Two-dimensional gas of massless Dirac fermions in graphene. Nature 438, 197-200 (2005).

28. Zhang, Y. B., Tan, Y. W., Stormer, H. L. \& Kim, P. Experimental observation of the quantum Hall effect and Berry's phase in graphene. Nature 438, 201-204 (2005).

29. Kravets, V. G., Schedin, F. \& Grigorenko, A. N. Plasmonic blackbody: almost complete absorption of light in nanostructured metallic coatings. Phys. Rev. B 78, 205405 (2008).

30. Schedin, F. et al. Detection of individual gas molecules adsorbed on graphene. Nat. Mater. 6, 652-655 (2007)

31. Das, A. et al. Monitoring dopants by Raman scattering in an electrochemically top-gated graphene transistor. Nat. Nanotechnol. 3, 210-215 (2008).

32. Basko, D. M., Piscanec, S. \& Ferrari, A. C. Electron-electron interactions and doping dependence of the two-phonon Raman intensity in graphene. Phys. Rev. B 80, 165413 (2009).

33. Horng, J. et al. Drude conductivity of Dirac fermions in graphene. Phys. Rev. B 83, 165113 (2011).

34. Maier, S. A. \& Atwater, H. A. Plasmonics: localization and guiding of electromagnetic energy in metal/dielectric structures. J. Appl. Phys. 98, 011101 (2005).

35. Kravets, V. G. et al. Cascaded optical field enhancement in composite plasmonic nanostructures. Phys. Rev. Lett. 105, 246806 (2010).

36. Kravets, V. G., Schedin, F. \& Grigorenko, A. N. Extremely narrow plasmon resonances based on diffraction coupling of localized plasmons in arrays of metallic nanoparticles. Phys. Rev. Lett. 101, 087403 (2008).

\section{Acknowledgements}

We are grateful to the Royal Society (UK), Engineering and Physical Research Counci (UK), European Research Council, EU grants Rodin and Marie Curie ITN-GENIUS (PITN-GA-2010-264694), a SAIT GRO Program and Nokia Research Centre (Cambridge) for financial support, and to Elefterios Lidorikis for useful discussions.

\section{Author contributions}

K.S.N. and A.K.G. conceived the project. K.S.N., A.C.F. and A.N.G. designed the experiment. T.J.E. and R.V.G. created the samples. T.J.E., L.B., P.K.J., A.L. and K.S.N. developed the measurement setup and performed the measurements. A.N.G. has done the modelling. All the authors participated in the discussion of the results and in the writing of the paper. The whole project was supervised by K.S.N, A.K.G., A.C.F. and A.N.G.

\section{Additional information}

Competing financial interests: The authors declare no competing financial interests.

Reprints and permission information is available online at http://npg.nature.com/ reprintsandpermissions/

How to cite this article: Echtermeyer, T. J. et al. Strong plasmonic enhancement of photovoltage in graphene. Nat. Commun. 2:458 doi: 10.1038/ncomms1464 (2011). 